\title{
Highlights from the X-ray Astronomy Satellite Hitomi (ASTRO-H)
}

\author{
Manabu Ishida*, on behalf of Hitomi Collaboration \\ Institute of Space and Astronautical Science/JAXA \\ 3-1-1 Yoshinodai, Chuo Ward, Sagamihara, Kanagawa 252-5210, Japan \\ E-mail: ishida@astro.isas.jaxa.jp
}

The 6th Japanese X-ray astronomy satellite Hitomi was successfully launched on 2016 February 17th. The main mission instrument SXS (the X-ray micro calorimeter) has achieved spectral resolution of $4.97 \mathrm{eV}$ at $5.9 \mathrm{keV}$ in orbit, which is much better than any CCD camera in orbit so far by more than 30 times. Although Hitomi's life in orbit is unfortunately only $\sim 1$ month, the SXS has high resolution spectroscopy for several targets. In the Perseus cluster observation, the SXS detected the turbulent motion of the ICM plasma for the first time, and found that the jet from the central AGN (NGC 1275) is not dynamically powerful enough to sustain the central part of the ICM as hot as it is; there must be some other heat sources. In the observation of N132D, the SXS discovered that the optically thin thermal plasma, traced with an iron He $\alpha$ emission line, is receding from us with a velocity of $1200 \mathrm{~km} \mathrm{~s}^{-1}$ in the rest frame of the host galaxy LMC. This result suggests that the progenitor explosion was highly anisotropic. In this way the SXS has demonstrated the power of non-dispersive high resolution spectroscopy. Accordingly, JAXA and NASA plan to jointly implement a recovery mission named XARM (X-ray Astronomy Recovery Mission), with the SXS, equivalent to that onboard Hitomi, as the main mission instrument. Currently the launch is planned to be made by the end of March 2021.

XII Multifrequency Behaviour of High Energy Cosmic Sources Workshop

12-17 June, 2017

Palermo, Italy

${ }^{*}$ Speaker. 


\section{Introduction to Hitomi and its Mission Instruments}

\subsection{The X-ray Astronomy Satellite Hitomi}

Hitomi [1], referred to as ASTRO-H prior to the launch, is the sixth Japanese X-ray astronomy satellite developed under a vast international collaboration among JAXA, NASA, ESA and CSA, and more than 200 scientists worldwide participated in its development, calibration, operation, and scientific activities. Figure 1 shows the configuration of the mission instruments onboard Hitomi. The net weight of the spacecraft is $2,750 \mathrm{~kg}$, and the electric power produced by the solar paddle

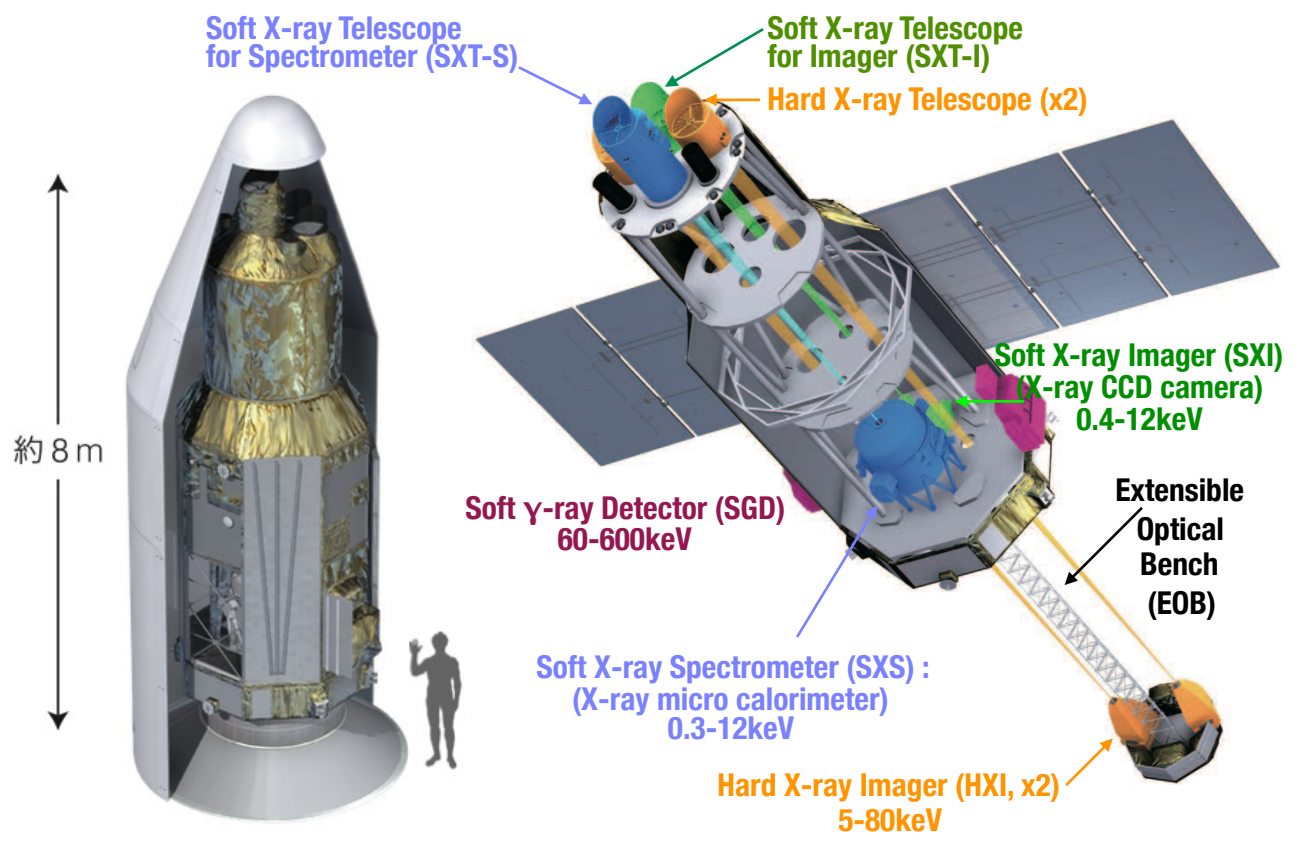

Figure 1: Configuration of the scientific instruments onboard Hitomi. The left sketch shows the spacecraft stowed in a fairing of JAXA's H-2A rocket.

array is about 3,500 W. At the time of the launch, the length of the spacecraft is some $8 \mathrm{~m}$. After extension of the optical bench in orbit, it becomes some $14 \mathrm{~m}$.

\subsection{Soft X-ray Spectrometer (SXS)}

The primary mission instrument of Hitomi is the Soft X-ray Spectrometer (SXS: [2]) which is placed at the on-axis focus of one of the Soft X-ray Telescopes (SXT-S: [3]). The SXS is a microcalorimeter that measures the energy of an incoming X-ray photon as increase of temperature of the detector. In order to sense a tiny energy of the single X-ray photon $\left(\sim 10^{-9} \mathrm{~J}\right)$, the detector is cooled as much as possible, since the heat capacity of a solid is proportional to $T^{3}$. In the case of Hitomi, the detector is deeply embedded into a dewar, and is cooled down to $\sim 50 \mathrm{mK}$. Figure 2 shows pictures of the detector array and outlook of the dewar, and an X-ray spectrum of 

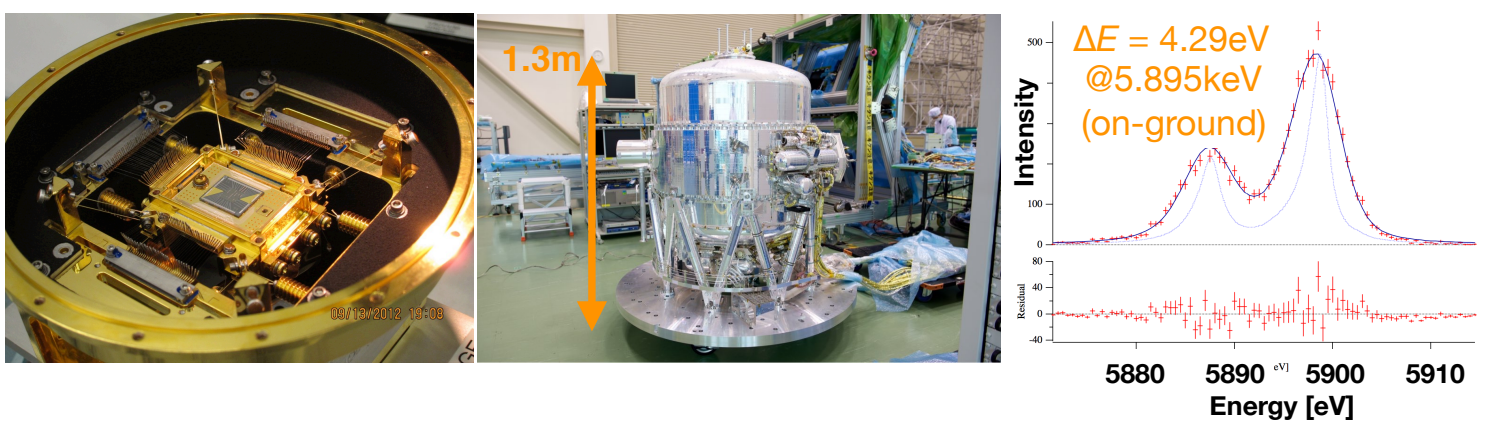

Figure 2: The SXS detector array (left), outlook of the SXS dewar (middle), and an X-ray spectrum of ${ }^{55} \mathrm{Mn}$ $\mathrm{K} \alpha$ line taken on-ground. The energy resolution of $4.3 \mathrm{eV}$ is achieved.

${ }^{55} \mathrm{Mn} \mathrm{K} \alpha$ line taken before the launch. The energy resolution $\Delta E$ is $4.3 \mathrm{eV}$, which is much better than conventional X-ray CCD cameras by more than 30 times. The SXS covers the energy band 0.3-12 keV, which includes K-shell emission line energies of all abundant elements in the universe.

Figure 3 shows the effective area and the energy-resolving power of the SXS versus the X-ray energy, in comparison with the XMM-Newton RGS and the Chandra LETG and HETG. One of the
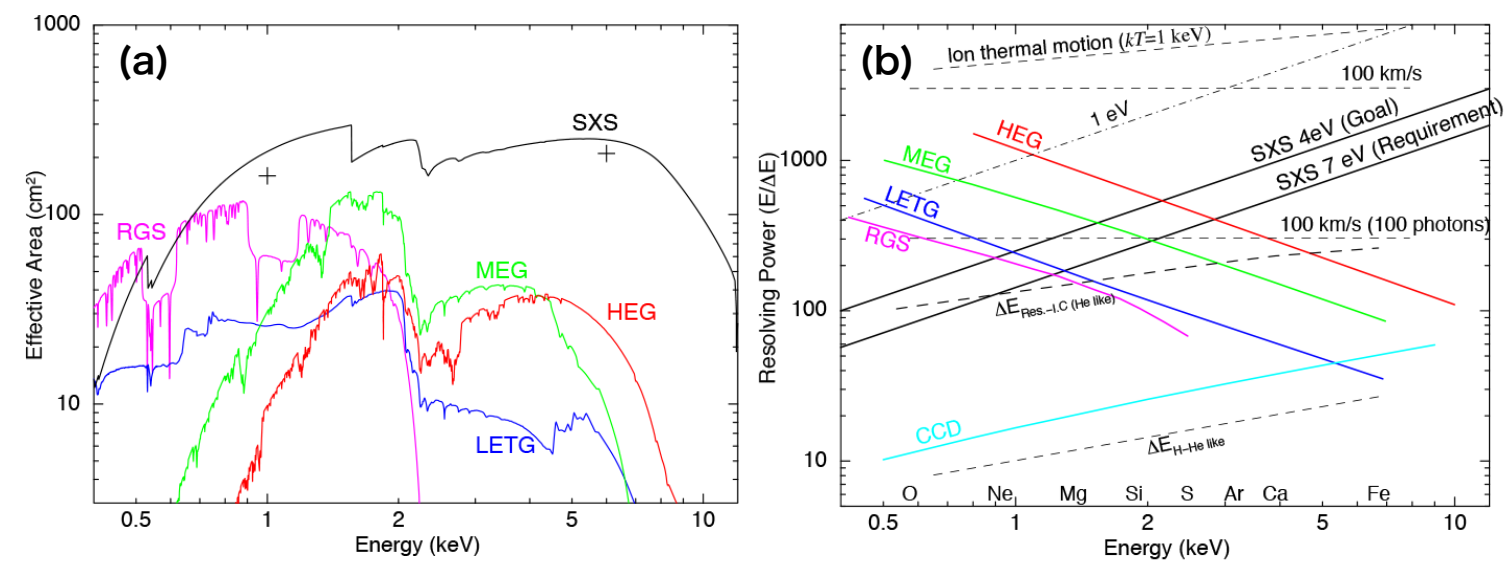

Figure 3: Effective area (a) and energy-resolving power of the SXS, in comparison with the other high resolution instruments onboard XMM-Newton and Chandra.

advantages of the SXS compared to the other high-resolution instruments is that the calorimeter is a non-dispersive instrument. This implies that the SXS can take for the first time a high-resolution spectrum of a wide-spread diffuse source, such as a cluster of galaxies. Moreover, above $\sim 2 \mathrm{keV}$, the effective are is larger by an order of magnitude and the energy-resolving power is better than the other high-resolution instruments. This means that the SXS can carry out a better K-line spectroscopy for the elements heavier than silicon. In particular, with the advent of the SXS, systematic high-resolution line spectroscopy of iron comes into view for the first time.

The detector of the SXS is composed of a $6 \times 6$ array of mercury telluride (HgTe) pixels each of which is a square with $818 \mu \mathrm{m}$ on a side. They are arranged with a pitch of $832 \mu \mathrm{m}$. Since the focal length of the SXT-S is $5.6 \mathrm{~m}$, the detector covers a square field of view with 3.06 arcmin on a side. 


\subsection{Other Observing Systems}

Hitomi is equipped with three more detecting systems. One is the Soft X-ray Imager (SXI: [4]) which adopts a conventional Charge-Coupled Device (CCD) and is located at the on-axis focus of the other soft X-ray telescope (SXT-I: [3]). Note that the two SXT modules, the SXT-S and the SXT-I, are identical in design. The SXI comprises of four CCD chips and forms a square detecting area with $63 \mathrm{~mm}$ on a side, which corresponds to 38.7 arcmin for the $5.6 \mathrm{~m}$ focal length of the SXTI. The SXI covers nearly the same energy band of $0.4-12 \mathrm{keV}$ as that of the SXS, and complements the narrow field of view of the SXS.

Another is the Hard X-ray Imager (HXI: [5]) located in the focal plane of the Hard X-ray Telescope (HXT: [6]). The HXT utilizes super mirror technology in which platinum/carbon multilayer is coated on the mirror foils. Owing to Bragg reflection on the super mirror surfaces, the HXT can collect X-ray photons from $5 \mathrm{keV}$ up to $80 \mathrm{keV}$. The detector HXI adopts cadmium-telluride (CdTe) chip which is a square with $32 \mathrm{~mm}$ on a side as an X-ray sensor. In order to measure two-dimensional positions of the X-ray photons reflected off the HXT, the chip is sandwiched by crisscross micro strip electrodes with a pitch of $0.25 \mathrm{~mm}$. The focal length of the HXT is $12 \mathrm{~m}$. Accordingly, the spatial resolution of the HXI is $4.30 \mathrm{arcsec}$ in principle. In order to retain enough observational efficiency, two modules of the HXT-HXI system are mounted onto the spacecraft. The system with such a long focal length, however, cannot be accommodated into the space of the fairing. Hence, as shown in Fig. 11 the HXI modules are mounted at the end of the Extensible Optical Bench (EOB: [7]), and were deployed after the launch.

The other is the Soft Gamma-ray Detector (SGD: [8]) which is composed of two equivalent modules adapted on side panels of the spacecraft body. The detector part comprises of four layers of silicone detectors and one layer of CdTe detector all of which are pixelized. Orientation of incoming $\gamma$-ray photon is measured by utilizing the Compton scattering among different layers. The SGD is sensitive to the X-ray/soft $\gamma$-ray photons in the band $60-600 \mathrm{keV}$.

Figure 4 summarizes the energy band covered by the mission instruments onboard Hitomi. With the four kinds of the observing systems, SXT-S/SXS, SXT-I/SXI, HXT/HXI, SGD, Hitomi

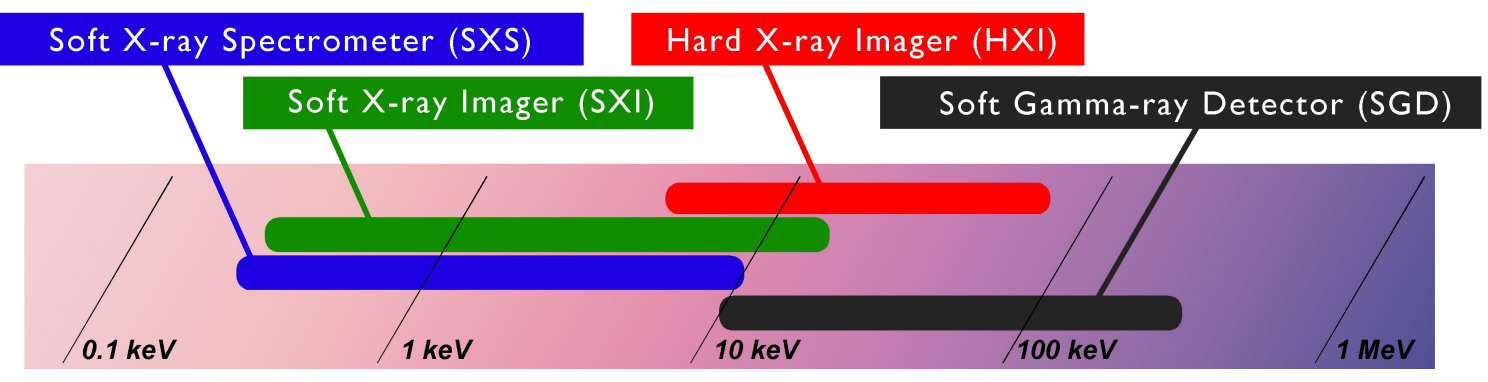

Figure 4: The energy band covered by the four mission intstruments onboard Hitomi

can cover the energy band $0.3-600 \mathrm{keV}$.

\section{Launch}

The X-ray astronomy satellite ASTRO-H was launched at 17:45(JST) on February 17th, 2016 from Tanegashima Space Center with JAXA's H-2A rocket. Figure 5 shows a picture of the launch 
scene. The launch was perfect and the spacecraft was thrown into an orbit with a perigee and an

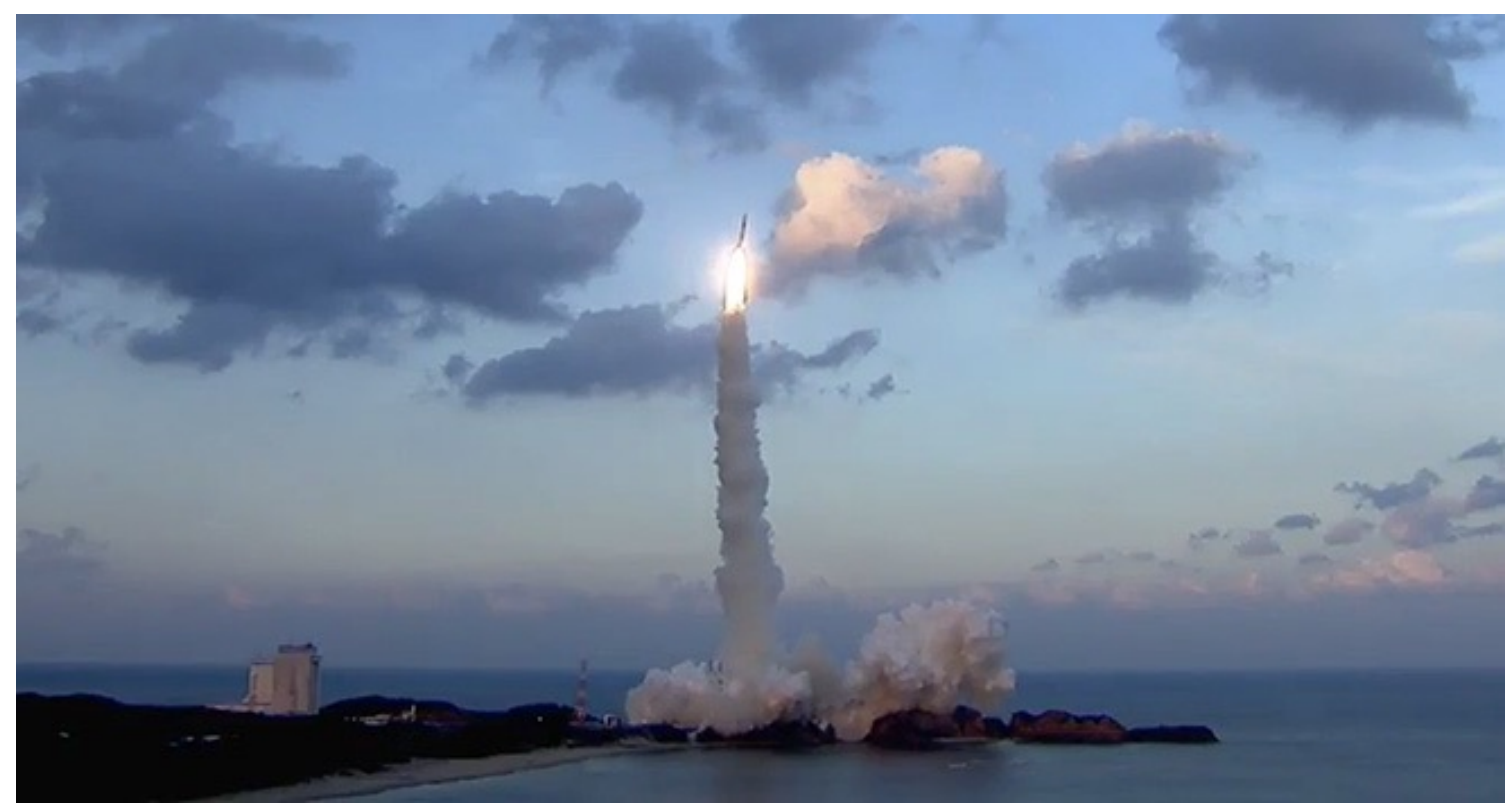

Figure 5: Launch!

apogee of $574.42 \mathrm{~km}$ and $576.52 \mathrm{~km}$, respectively, with an inclination angle of $30^{\circ} .998$. The orbital period is $96.2 \mathrm{~min}$.

\section{Observations}

Hitomi started observations of celestial objects on 2016 February 25, one week from the launch, after deployment of the solar array paddles, initial startup of the attitude and orbit controlling system (AOCS) and the primary instrument SXS. Unfortunately, Hitomi passed away on May 25 due to malfunction of the AOCS, Hitomi observed the following six targets during one month of its life: Perseus cluster, N132D (thermal SNR in the Large Magellanic Cloud: LMC), IGR J16318-4848 (highly obscured HMXB), G21.5-0.9 (non-thermal SNR), RX J1856.5-3754 (isolated neutron star), and Crab Nebula. All objects were pointed for 2-4 days. Some had, however, very short exposure time due to premature attitude parameter tuning or malfunction of common instruments. They include N132D and IGR J16318-4848, which had very limited SXS data because the spacecraft pointed away from the target at very early phases of the observations, and RX J1856.5-3754, which was out of the SXS field of view throughout the observation.

\section{Scientific Results}

The first bunch of scientific results from all mission instruments will be published in a special issue of Publication of Astronomical Society of Japan (PASJ) around the end of 2017. In this paper, however, we would like to concentrate on results of high-resolution spectroscopy carried out with the main instrument SXS. They came from the observations of Perseus cluster and N132D. The results from Perseus cluster described in the following subsections $\$ 4.1 .3$ and $\$ 4.1 .4$ have already 
been published in [9] and [10], respectively. That of N132D presented in $\$ 4.2$ will appear in the PASJ special issue [11].

\subsection{Perseus Cluster}

\subsubsection{Introduction}

Perseus cluster of galaxies is one of the X-ray brightest galaxy cluster locating at $z=0.01756$. The cluster is filled with hot gas with a temperature of $5 \times 10^{7} \mathrm{~K}$. The Chandra X-ray image obtained with a long exposure observation revealed there are inner bubbles which are associated with the bipolar jet of NGC1275 [12]. The location of the bubbles seems to coincide with radio emission sites.

There have been some questions raised as to the cluster hot gas. One is whether the central AGN is able to heat the central part of the intra cluster medium (ICM). It has been known that the cooling time of the ICM is shorter than the Hubble time at the cluster core. Hence, to maintain the cluster hot gas as it is, some heat source should exist. As the central part of the Perseus cluster has a complicated, non-uniform structure, associated with the bipolar jet of NGC1275, a turbulent motion of the hot gas has been expected. The turbulence triggered by jets of the central AGN can be one of the plausible candidates of the heat source. Another question is how much the turbulent motion contribute to the total kinetic energy of the ICM. The turbulent motion is essentially important to evaluate the amount of the dark matter which forms a gravitational well and traps the ICM, because its mass is evaluated under the assumption of hydrostatic equilibrium of the ICM with the gravitational potential of the dark matter. If the turbulent motion is non-negligible compared with the thermal motion, the evaluation of dark matter mass should be increased accordingly.

\subsubsection{In orbit Performance of the SXS}

In order to answer these questions, Hitomi selected Perseus cluster as the first science target of the mission. The observation of Perseus cluster was carried out during February 25-27 and March 4-6, 2016 for $290 \mathrm{ksec}$ in total. The left panel of Fig. 6 shows the SXS field of view (orange) during the observation of Perseus cluster handled in this proceeding, overlaid on the long-exposure Chandra image. The SXS covers a square region with $200 \mathrm{k}-\mathrm{ls}$ on a side including the central
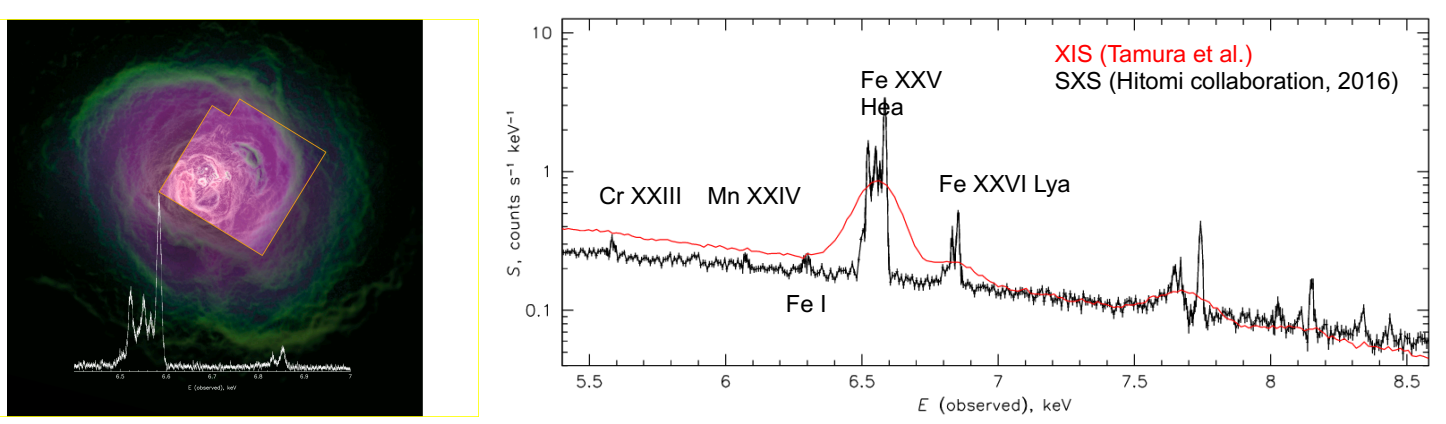

Figure 6: Left: The SXS field of view during the observation of Perseus cluster overlaid on the longexposure Chandra image, right: The SXS spectrum of Perseus cluster in the band 5.4-8.6 keV, overlaid with the spectrum of the Suzaku CCD camera (XIS) in red color after [13]. 
AGN (NGC 1275). The right panel shows the corresponding spectrum in the iron and nickel Kshell emission region. A spectrum taken with the Suzaku XIS (CCD camera) is overlapped in red color. The in-flight energy resolution of the SXS at ${ }^{55} \mathrm{Mn}-\mathrm{K} \alpha$ line energy $(5.895 \mathrm{keV})$ is $4.97 \mathrm{eV}$. With this superior energy resolution, the SXS can resolve He $\alpha$ triplet of iron clearly for the first time.

\subsubsection{Turbulence velocity at the center of the Perseus cluster}

Figure 7 shows an SXS spectrum at around the energy of Fe He $\alpha$ line which is extracted from the outer region drawn in red color in the left panel [9]. The green curve in the spectrum (right
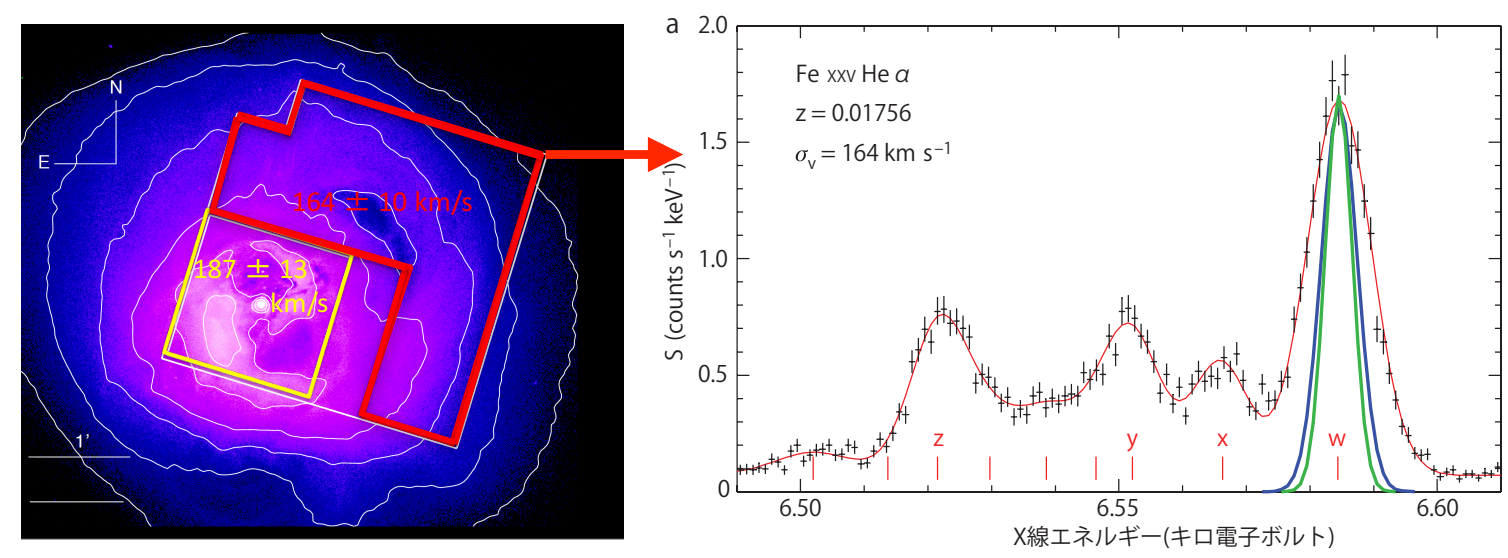

Figure 7: The right panel shows the spectrum of an outer region of the SXS in the band 6.49-6.56 keV 9 . The sky region adopted to extract this spectrum is shown in the left panel. The green and blue curves are the line spread functions of the resonance (w) component inherent to the detector $(\Delta E=4.97 \mathrm{keV}$ at Mn-K $\alpha$ line) and that accounts for the thermal broadening effect (about $80 \mathrm{~km} \mathrm{~s}^{-1}$ for iron), respectively. The red curve is the best-fit model to the entire $\mathrm{Fe} \mathrm{He} \alpha$ line which further takes into account the turbulent velocity of $164 \mathrm{~km} \mathrm{~s}^{-1}$.

panel) shows the line profile of the resonance component (w) only with an intrinsic width of the detector ( $\Delta E=4.97 \mathrm{keV}$ at ${ }^{55} \mathrm{Mn}-\mathrm{K} \alpha$ line). The blue curve accounts for the thermal motion of iron for the w-component $\left(80 \mathrm{~km} \mathrm{~s}^{-1}\right)$. The red curve further adds a velocity dispersion of $164 \mathrm{~km} \mathrm{~s}^{-1}$, which can successfully fit the entire $\mathrm{Fe} \mathrm{He} \alpha$ spectrum. This velocity dispersion is interpreted as originating from turbulent motion of the plasma. Even if so, however, the pressure originating from the turbulent motion is only $4 \%$ of the thermodynamic pressure. As a result, the dark matter mass estimation based on the hydrostatic assumption does not need major change.

We have carried out quite similar analysis to the central region (yellow color in the left panel of Fig. (7), and derived a slightly larger velocity dispersion of $183 \pm 13 \mathrm{~km} \mathrm{~s}^{-1}$. This can be interpreted that the central AGN stimulates the turbulent motion. Nevertheless, it is found difficult to heat and maintain the central part of the ICM as it is through the turbulent motion. There must be some other heat source that prevents the central part of the ICM from cooling rapidly.

\subsubsection{The 3.5 keV emission line of the Perseus cluster}

Another interesting topic associated with Perseus cluster is a putative emission line at $\sim 3.5 \mathrm{keV}$, which was first reported by Bulbul et al. 14] based on an integrated spectrum of 73 clusters ob- 
served with XMM-Newton. Since then the origin of this line has been a matter of debate, and one possible origin is the decay of the "sterile neutrino". If so, its mass should be $\sim 7.1 \mathrm{keV}$. The same authors reported similar detection from some other sky regions of Perseus clusters. It is, however, not very easy to establish the detection of the $3.5 \mathrm{keV}$ line, because of a limited energy resolution of the CCD cameras in orbit. For example, positive detections ([15], [16]) and an upper limit ([17]) were both reported from the same Suzaku data.

We have therefore attempted to analyze the SXS data around $3.5 \mathrm{keV}$ to finally resolve the issue [10]. As noted in $\S 1.2$, however, the field of view of the SXS is only 3.06 arcmin. Accordingly, we have first integrate the XMM-Newton MOS spectrum from the same sky region as that of the SXS. The $3.5 \mathrm{keV}$ line flux in this sky region has turned out to be $9.0 \pm 2.9 \times 10^{-6} \mathrm{ph} \mathrm{s}^{-1} \mathrm{~cm}^{-2}$, which means the line in the SXS field of view is detected at a confidence level of only $\sim 3 \sigma$. We then have extracted an SXS spectrum from the SXS data. The spectrum in the band 2.9-4.1 keV is shown in Fig 8 a). In this panel, the red and blue bars below the spectrum indicate the allowed energy ranges
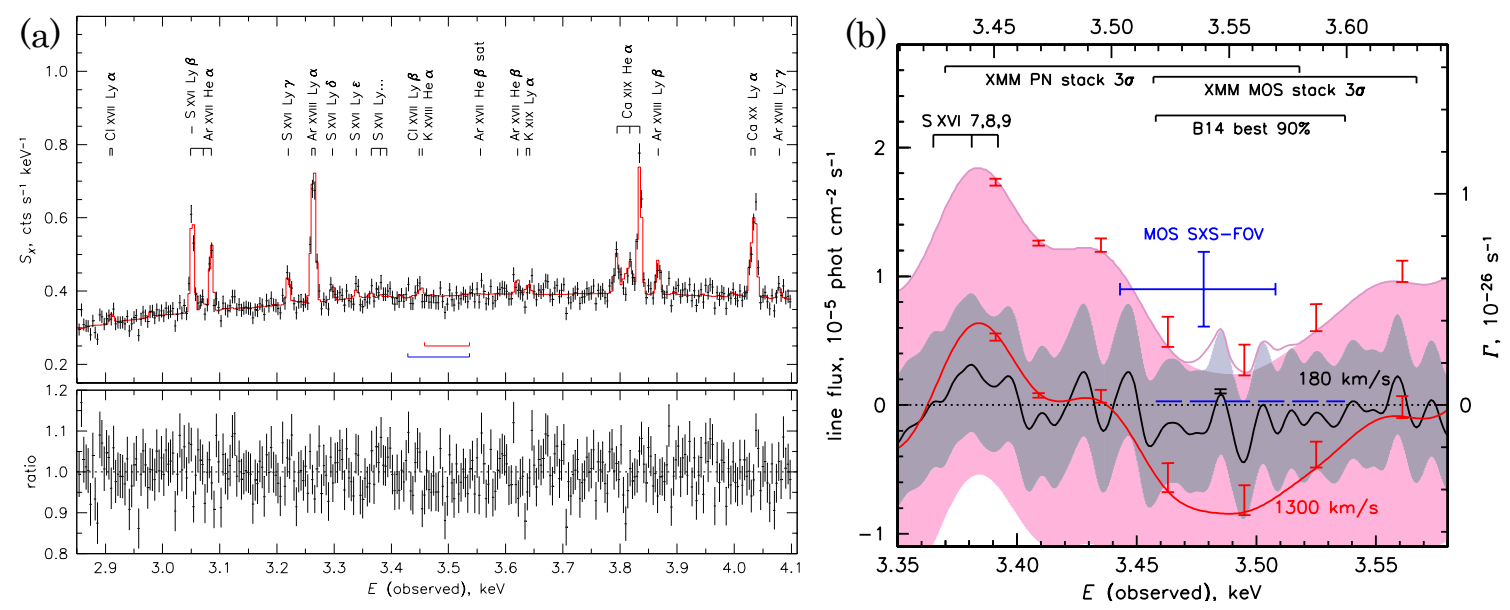

Figure 8: (a) The SXS spectrum of Perseus cluster in the band 2.9-4.1 keV. The best-fit model comprising of a thermal and power-law components with a line-of-sight velocity dispersion of $\sigma=180 \mathrm{~km} \mathrm{~s}^{-1}$ is overlaid in red color. The red and blue bars below the spectrum are the allowed energy ranges derived from the MOS data integrated from the 73 clusters and from Perseus cluster, respectively. (b) Blind line search result with the SXS data. Black and red colors are used for trial lines with a line-of-sight velocity dispersions of $180 \mathrm{~km} \mathrm{~s}^{-1}$ and $1300 \mathrm{~km} \mathrm{~s}^{-1}$, respectively. The blue cross is the allowed range from the MOS data at the $1 \sigma$ confidence level.

of the $3.5 \mathrm{keV}$ line derived from the MOS data integrated from the 73 clusters and solely from Perseus cluster, respectively. At first glance, there is no sign of the emission line found in these ranges. A more quantitative results is shown in Fig.8 (b), where the flux upper limit (emission) and the lower limit (absorption) at a confidence level of $3 \sigma$ are show at trial line energies from $3.35 \mathrm{keV}$ to $3.58 \mathrm{keV}$. The black and red colors are used for a line with a velocity dispersion of $180 \mathrm{~km} \mathrm{~s}^{-1}$ and $1300 \mathrm{~km} \mathrm{~s}^{-1}$. The former agrees with the turbulent velocity derived in the previous subsection while the latter is consistent with that predicted from the velocity dispersion of the component galaxies. We conclude from Figure 8 (b) that Hitomi did not detect the $3.5 \mathrm{keV}$ emission line and that it is not so strong as the XMM-Newton MOS detected even if it really exists. 


\subsection{N132D}

N132D is the X-ray brightest SNR in the LMC. Its oxygen-rich X-ray spectrum indicates that N132D is a remnant of a core-collapsed supernova exploded some 2500 years ago. The Suzaku spectrum is characterized by a strong $\mathrm{Fe} \mathrm{He} \alpha$ line, and suggests that the plasma is recombining with parameters of $k T_{\text {init }} \simeq 10 \mathrm{keV}, k T_{\mathrm{e}} \simeq 2 \mathrm{keV}$, and $n_{\mathrm{e}} t \simeq 5 \times 10^{11} \mathrm{~cm}^{-3} \mathrm{~s}$ [18]. Due to its brightness, rich emission lines, and a moderate spatial extent of $\sim 2$ arcmin, being well fitted into the SXS field of view, N132D is selected as one of the initial phase targets of the SXS. Unfortunately, however, due to malfunction in the AOCS system, only $3.7 \mathrm{ksec}$ data are available with the SXS, and only 17 photons are obtained in the Fe He $\alpha$ band. Nevertheless, the high spectral resolution of the SXS enables us to do some science with this small number of photons. Figure 9 (a) shows the spectrum in the band 6.45-6.80 keV that fully includes the photons of the $\mathrm{Fe} \mathrm{He} \alpha$ line, overlaid with an optically thin thermal model with no line-of-sight velocity that provides with the best-fit to the spectrum [11]. This panel demonstrates that, although the continuum was scarcely detected due to

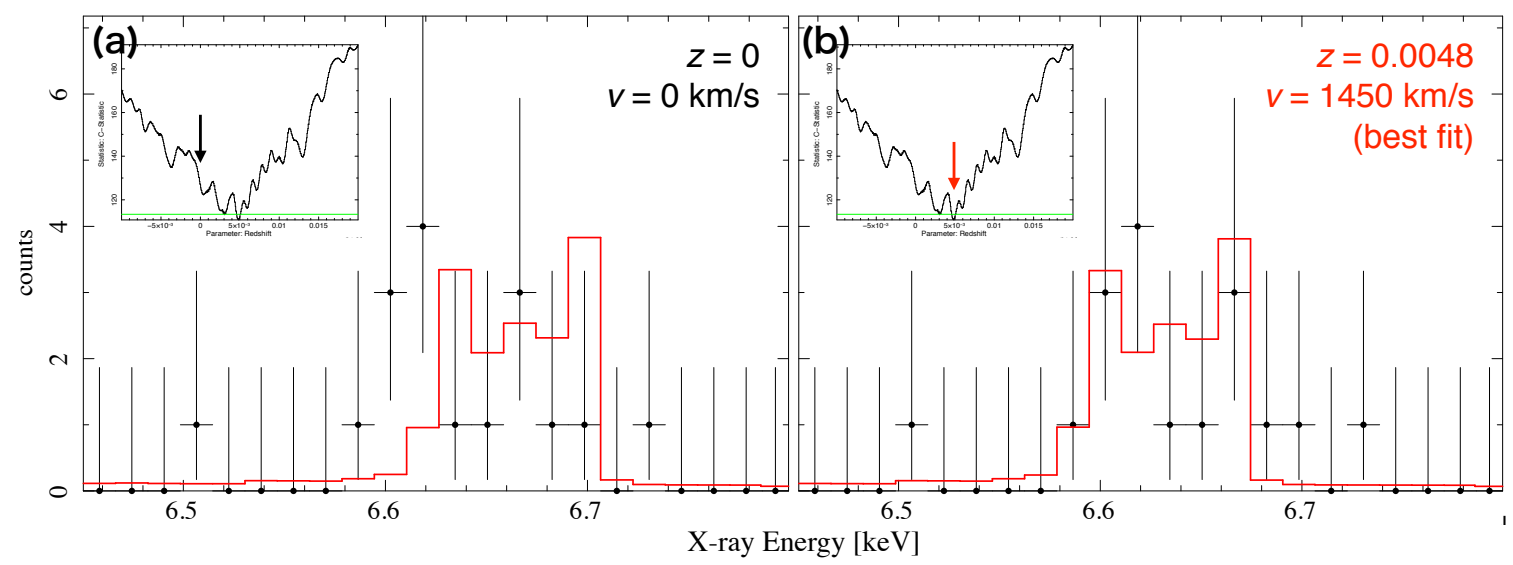

Figure 9: The SXS spectrum of N132D overlaid with a best-fit optically thin thermal model with (a) $z=0$ and (b) $z=0.0048$. The insets are C-statistics diagrams versus trial redshift.

the short exposure time, the line sensitivity is extremely high thanks to the high spectral resolution. Figure 9 a) further suggests that the detected photons somewhat shift from the best-fit $z=0$ model in the lower energy side. As a matter of fact, the C-stat diagram (see the inset) indicates there is a significant red-shift of the emission lines. The spectrum and the model that gives the best fit with varying the redshift is shown in Fig. 9 b). The best-fit $z=0.0048$ implies a recessional velocity of $1450 \mathrm{~km} \mathrm{~s}^{-1}$. This exceeds the recessional velocity of the LMC itself $275 \mathrm{~km} \mathrm{~s}^{-1}$. In the rest frame of the LMC, the N132D plasma is receding from us with a velocity of $1200_{-700}^{+300} \mathrm{~km} \mathrm{~s}^{-1}(90 \%$ confidence level). This probably suggests that the explosion of the progenitor was anisotropic. The result described in this subsection will appear in [11].

\section{Summary and Future Prospect}

Hitomi is the 6th Japanese X-ray astronomy satellite and was successfully launched on 2016 February 17th from Tanegashima Space Center with the 30th H-2A rocket. The main mission instrument SXS has achieved spectral resolution of $4.97 \mathrm{eV}$ at $5.9 \mathrm{keV}$, which is much better than 
any CCD camera in orbit by more than 30 times. Unlike XMM-Newton's RGS and Chandra's LETG/HETG, the SXS is a non-dispersive detector, and hence enables to carry out fine spectroscopy for a diffuse source in general for the first time. Moreover, the SXS has the largest effective area among these high resolution detectors, and the best energy resolution above $\sim 2 \mathrm{keV}$. With these unique characteristics, we can do a high resolution spectroscopy of K-shell emission lines, especially those from iron.

Unfortunately Hitomi's life in orbit is only about 1 month, the SXS has demonstrated its power of high resolution spectroscopy. In the Perseus cluster observation, the SXS detected the turbulent motion of the ICM plasma for the first time, and found that the turbulent pressure is only about $4 \%$ of the thermal pressure. The jet from the central AGN (NGC1275) is not powerful enough to sustain dynamically the central part of the ICM as hot as it is; there must be some other heat sources. In the observation of N132D, the SXS discovered that the optically thin thermal plasma is receding from us with a velocity of $1450 \mathrm{~km} \mathrm{~s}^{-1}$, which is much larger than that of the host galaxy LMC itself. This result suggests that the progenitor explosion was highly anisotropic.

Although the life of Hitomi was very short, it is demonstrated that the SXS is able to open up a new field window of the X-ray astronomy in terms of a non-dispersive high resolution spectroscopy. Accordingly, JAXA and NASA plan to jointly implement a recovery mission named XARM (Xray Astronomy Recovery Mission), with the SXS as the main mission instrument. An X-ray CCD camera, equivalent to Hitomi's SXI, will also be onboard. The launch is currently planned by the end of March 2021.

\section{References}

[1] Takahashi, T., Kokubun, M., Mitsuda, et al., The ASTRO-H (Hitomi) X-ray Astronomy Satellite, JATIS (2018) submitted

[2] Kelley, R., et al., The ASTRO-H high-resolution soft x-ray spectrometer, JATIS (2018) submitted

[3] Serlemitsos, P. J., Okajima, T., Soong, J., et al., Design and Fabrication of ASTRO-H (Hitomi) Soft X-ray Telescope - Aluminum Foil X-ray Mirror —, JATIS (2018) submitted

[4] Tanaka, T., Uchida, H., Nakajima, H., et al., The Soft X-ray Imager (SXI) aboard the Hitomi Satellite, JATIS (2018) submitted

[5] Nakazawa, K., Sato, G., Kokubun, M., et al., The hard X-ray imager (HXI) onboard the Hitomi (ASTRO-H) Satellite, JATIS (2018) submitted

[6] Awaki, H., Kunieda, H., Ishida, M., et al., Hard $x$-ray telescopes to be onboard Hitomi (ASTRO-H), Applied Optics 53 (2014) 7664

[7] Ishimura, K., Ishida, M., Kawano, T., et al., Induced Vibration of High-Precision Extensible Optical Bench during Extension on Orbit, Tr. Japan Soc. Aero. Sp. Sci (2017) submitted

[8] Tajima, H., Watanabe, S., Fukazawa, Y., et al., Design and Performance of Soft Gamma-ray Detector onboard the Hitomi (ASTRO-H) Satellite, JATIS (2018) submitted

[9] Hitomi Collaboration, Aharonian, F., Akamatsu, H., et al., The quiescent intracluster medium in the core of the Perseus cluster, Nature 535 (2016) 117

[10] Aharonian, F. A., Akamatsu, H., Akimoto, F., et al., Hitomi Constraints on the $3.5 \mathrm{keV}$ Line in the Perseus Galaxy Cluster, Ap. J. Lett. 837 (2017) L15 
[11] Hitomi Collaboration, Aharonian, F., Akamatsu, H., et al., Hitomi Observations of hte LMC SNR N132D: Highly Redshifted X-ray Emission from Iron Ejecta, JATIS (2018) submitted

[12] Fabian, A. C., Sanders, J. S., Allen, S. W., et al., A deep Chandra observation of the Perseus cluster: shocks and ripples, MNRAS 344 (2003) L43

[13] Tamura, T., Maeda, Y., Mitsuda, K., et al., X-ray Spectroscopy of the Core of the Perseus Cluster with Suzaku: Elemental Abundances in the Intracluster Medium, Ap. J. Lett. 705 (2009) L62

[14] Bulbul, E., Markevitch, M., Foster, A., et al., Detection of an Unidentified Emission Line in the Stacked X-Ray Spectrum of Galaxy Clusters, Ap. J. 789 (2014) 13

[15] Urban, O., Werner, N., Allen, S. W., et al., A Suzaku search for dark matter emission lines in the X-ray brightest galaxy clusters, MNRAS 451 (2015) 2447

[16] Tamura, T., Iizuka, R., Maeda, Y. et al., An X-ray spectroscopic search for dark matter in the Perseus cluster with Suzaku, PASJ 67 (2015) 23

[17] Franse, J., Bulbul, E., Foster, A., et al.,Radial Profile of the $3.5 \mathrm{keV}$ Line Out to R200 in the Perseus Cluster, Ap. J. 829124

[18] Bamba, A., et al. Ap. J (2017) submitted 